\title{
A simple index to predict myocardial infarction size and adverse clinical outcome in patients with acute STEMI undergoing primary PCI
}

\author{
Osama Amin Abd-Elhamid, Wael Samy, Akram Abdelbary , Tarek Elgohary , Alia Abd-El- \\ Fattah. \\ Critical Care Department, Faculty of Medicine, Cairo University \\ Corresponding author: email: Osama Amin Abd-Elhamid,email: osamaamin130@yahoo.com
}

\section{Abstract}

Background: Early improvement of perfusion after acute MI will improve left ventricle function and decrease the infarction area, thus decreasing mortality.

Methodology: 52 patients presented with acute ST segment elevation MI Who underwent primary PCI within 24 hours of presentation. All patients were subjected to full history taking, physical examination, serial ECG, cardiac enzymes, calculation of relative importance index (RII) by dividing culprit segment diameter by left anterior descending, circumflex and right coronary arteries at their proximal segments and myocardial perfusion image to detect infarction size.

Results: There is significant correlation between RII and left ventricular dysfunction

(p: 0.028). Significant correlation between RII and mortality are present.

Conclusion: RII is significantly correlated with adverse clinical outcome in patients with acute STEMI.

Key words: Simple index, STEMI, Primary PCI.

\section{Introduction}

Acute myocardial infarction (MI) indicates irreversible myocardial injury resulting in necrosis of a significant portion of the myocardium secondary to occlusion of an epicardial artery. The area of infarct occurs in the distribution of the occluded artery, which typically starts at the subendocardium and progresses towards the epicardium in a wavefront fashion ${ }^{(l)}$. Clinical outcome after acute MI depends largely on final infarct size and is improved with early reperfusion. The major determinant of final infarct size for a given coronary occlusion is the size of the myocardial area-at-risk $(\mathrm{AAR})^{(2,3)}$. Therefore, deter- mination of both initial AAR and final infarct size after acute MI has major clinical implications since it permits an accurate assessment of myocardial salvage provided by reperfusion therapies. Final infarct size is always less than the initial AAR.

Successful rescue PCI within 3-24 hours of the onset of chest pain has been associated with improved LV systolic function at a mean follow-up period of 22 months ${ }^{(4) .}$ In this study, baseline and repeated Echocardiography were used to assess LV systolic function. Other studies of primary PCI have also reported improved LV systolic function compared to thrombolysis ${ }^{(5)}$.

Early improvement of perfusion after MI will improve left ventricle function and decrease the infarction area, thus decreasing mortality $^{(6)}$ The efficacy of reperfusion treatment may be shown indirectly with electrocardiography (ECG), by regression of ST elevation, but there is a need for methods to demonstrate left ventricle and microvascular function improvement (7) .Primary percutaneous coronary intervention (PCI) is regarded as the best reperfusion model in STEMI.

Over the last 20 years, nuclear cardiology has become a mainstay in the evaluation of ischemic heart disease. In the setting of acute coronary syndromes (myocardial infarction or unstable angina), myocardial perfusion imaging has emerged as an important tool in assessing the functional significance of angiographic coronary stenosis, evaluating the efficacy of therapeutic intervention and risk-stratifying patients in the post infarction period ${ }^{(8)}$.

Myocardial perfusion imaging possesses not only diagnostic but also prognostic value, because it permits stratification of patients into categories of risk for future cardiac events ${ }^{(9-10)}$.

Patients and methods:

52 patients were prospectively enrolled in our study presented with their first STEMI event and were undergone primary PCI within the first 24 hours of presentation. The study was approved by the Ethics Board of Cairo University. 
We excluded patients with previous ischemic events, cardiogenic shock, severe valvular heart disease and patients with refractory ventricular arrhythmias.

All patients were subjected to full history taking regarding risk factors for ischemic heart disease as diabetes mellitus, hypertension, hyperlipidemia, any history of other diseases or regular medications.

Serial ECG was recorded, cardiac enzymes were measured, other biochemical parameters were also measured as serum creatinine, electrolytes, CBC and coagulation profile.

\section{Primary PCI procedure:}

$>$ The procedure was performed according to standard practice.

> Un-fractionated heparin 70U/kg was used for procedural anticoagulation.

$>$ Both direct stenting protocol and predilatation were included.

\section{Angiographic data:}

$>$ Angiographic data were collected including lesion location, reference vessel diameter, and severity of the culprit lesion, presence of thrombus and the presence of multi-vessel involvement. Angiographic assessment of coronary reperfusion was assessed using TIMI flow grade.

\section{Quantitative coronary analysis:}

$>$ All angiographic images were obtained with a digitalflat-panel cardiac imaging system (Allura Xper FD 20, Philips Medical Systems).

$>$ Analysis was performed by validated and automated edge-detection software (Inturis, Philips Medical Systems) in all patients in two orthogonal views. Coronary end-diastolic frames from views without shortening or overlap were selected for QCA measurement. Care was exercised to choose a projection where the vessel and the catheter tip run in a plane nearly parallel to the X-ray tube. Calibration was performed by contrastfilled $6 \mathrm{~F}$ diagnostic catheter of the same manufacturer (Boston Scientific Corporation; Natick, MA, USA) as a scaling device. The proximal diameters of the major coronary vessels (LAD, left circumflex artery (LCX) and right coronary artery (RCA) were measured in all patients. The proximal segment of the LAD and LCX was defined as vessel segment immediately beyond the bifurcation of the left main coronary artery (LMCA). In case of any disease in this segment, the first disease-free segment before the major bifurcation of the individual vessel was used for measurement. When LAD is ostially occluded thereby precluding measurement, we used an appropriately inflated stent size as a surrogate of the LAD diameter. The proximal segment of the RCA was defined as the vessel segment 1 to $2 \mathrm{~cm}$ distal to the coronary ostium before any major branch take-off except for conus branch. Patients with diffuse disease at proximal segments of coronary arteries that precluded defining a reference segment were excluded from the study. The culprit segment diameter was measured at the first disease-free reference segment before the occlusion. Total coronary artery diameter was defined as the sum of the diameters of LAD, LCX and RCA. RII for the culprit lesion was calculated by dividing the culprit segment diameter by the total coronary artery diameter.

\section{Myocardial Perfusion Imaging:}

Acquisition of SPECT images was performed within $1 \mathrm{~h}$ of the injection of the Tc-99m SestaMIBI using dual head Siemens gamma camera (Symbia E) utilizing Cedar Sinai software 1994-2009 (8 frames per cycle) Images were gated to the R wave of the ECG, and image acquisition was interrupted for one beat if the R-R interval varied by $15 \%$ of the preceding $\mathrm{R}-\mathrm{R}$ interval, equipped with a lowenergy, high-resolution parallel-hole collimators. Sixty-four projections were acquired in a $64 \times 64$ matrix over a $180^{\circ}$ from the $45^{\circ}$ right anterior oblique projection to the $45^{\circ}$ left posterior oblique projection. Images were gated at 8 frames per cardiac cycle. Processing was performed using a Back projection filter. All patients underwent 1-day rest imaging with administration of 25- 30 $\mathrm{mCi}$ Tc99m sestamibi (MIBI) with SPECT imaging performed 1 hour after injection. 


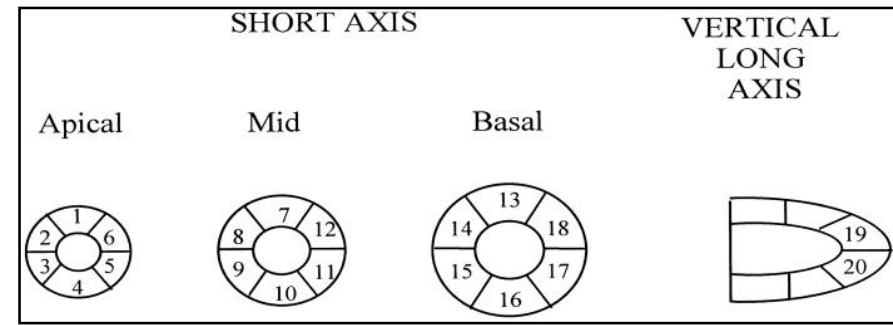

Figure (1): LV segmentation using 20segments model ${ }^{(11)}$.

The tracer uptake was analyzed on computer-generated polar map using twenty segment models. In the perfusion analysis, the polar maps of each patient were compared on a pixel-by-pixel basis Pixels with MIBI activity greater than 2.5 SDs below the corresponding normal mean values were considered abnormal. A perfusion defect was automatically expressed by the computer as the number of abnormal pixels divided by the total number of LV pixels $x$ 100. Rest myocardial segmental perfusion was scored on a semi quantitative five-point score scale of 0 to 4 ; where $0=$ normal, $1=$ =quivocal, $\quad 2=$ =moderate,$\quad 3=$ severe reduction of segment perfusion (radioisotope uptake) and $4=$ totally absent perfusion with absence of radioisotope uptake.

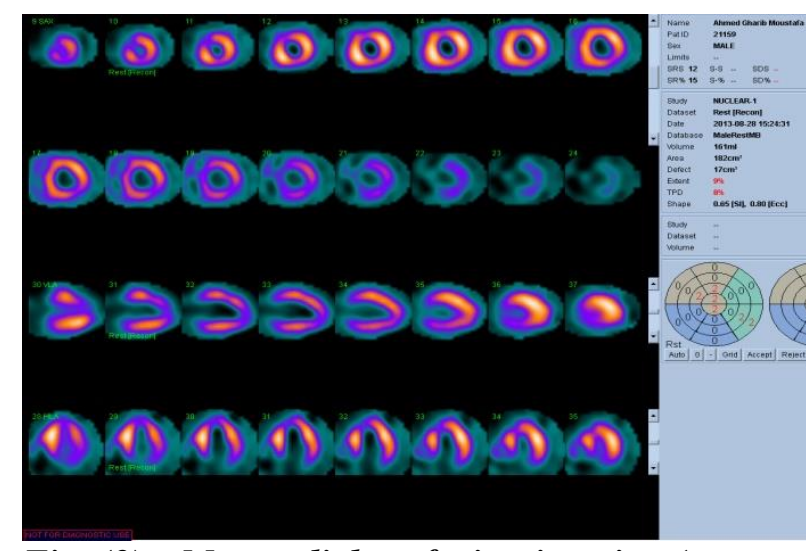

Fig. (2): Myocardial perfusion imaging (rest study) in patient number 14 with anterior MI with defect percentage $20 \%$

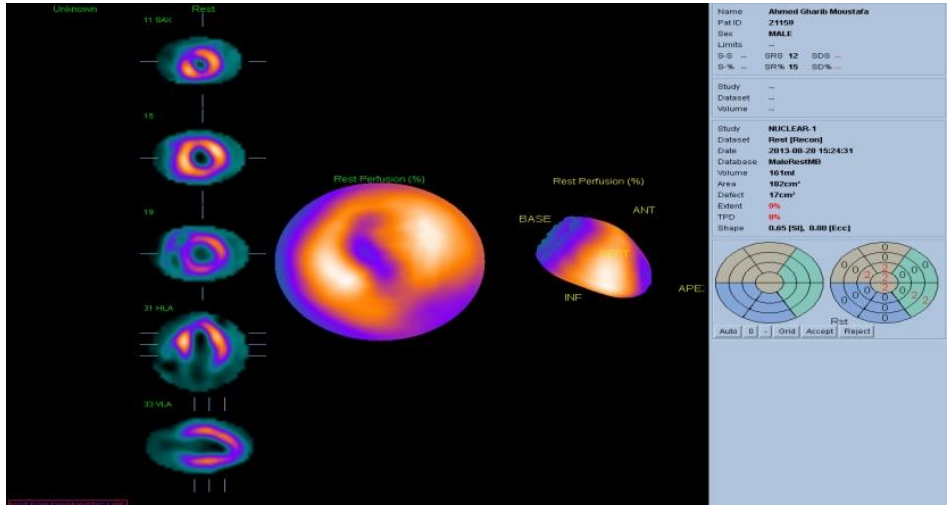

Fig. (3): Myocardial perfusion imaging (rest study) in patient number 14 with anterior MI with defect percentage $20 \%$

\section{- Echocardiography:}

All patients underwent echocardiographic examination. Each patient was examined in the left lateral position according to the recommendations of the American Society of Echocardiography (ASE). The study was conducted using an ATL HDI 5000 colored echocardiographic machine using a $3.5 \mathrm{MHz}$ transducer to assess LV systolic function.

Clinical Follow up:

- Chet pain resolution.

- Serial ECG.

- Serial cardiac enzymes.

- Major Adverse Cardiovascular End points (MACE) including: life threatening arrhythmias, cardiogenic shock, reinfarction, CVS and Mortality.

Statistical analysis:

Numerical data were analyzed as mean + SD. Categorical data were analyzed as percentages .Paired comparisons were done to compare pre and post PCI recordings. Statistical analysis was done using SPSS 17.0 edition.

\section{Results:}

Demographic data of this study included 44 male and 8 female, the age of patients ranged from 30 to 71 years .

Table (1): Distribution of the studied cases as regards clinical risk factors:

\begin{tabular}{|l|l|l|}
\hline Risk factors & No. & \% \\
\hline Dyslipidemia & 40 & $76.9 \%$ \\
\hline Diabetic & 15 & $28.8 \%$ \\
\hline HTN & 20 & $38.5 \%$ \\
\hline FH & 17 & $32.7 \%$ \\
\hline Smoking & 40 & $76.9 \%$ \\
\hline
\end{tabular}

The time of presentation was varying from 5 to 14 hours, ( $8.7 \pm 2.3$ hours). 
Table (2): Distribution of the studied cases as regards Culprit vessel:

\begin{tabular}{|l|l|l|}
\hline Culprit vessel & Frequency & Percentage \\
\hline LAD & 28 & $53.8 \%$ \\
\hline LCX & 9 & $17.3 \%$ \\
\hline RCA & 15 & $28.8 \%$ \\
\hline
\end{tabular}

Table (3): Simple QCA Index data of the present study:

\begin{tabular}{|l|l|l|l|}
\hline & Mean & SD & Median \\
\hline Simple QCA index & 0.328 & 0.036 & 0.328 \\
\hline
\end{tabular}

The minimum Simple QCA Index calculated pre-PCI was 0.265 and the maximum was 0.398 with mean value of 0.328 and standard deviation of 0.036 .

Considering cutoff 0.32 for MACE or heart failure, yielded sensitivity of $66.7 \%$ and specificity of $44.5 \%$. The present study results showed statistically significant relationship between simple QCA Index and systolic function of the left ventricle estimated by the ECHO ( P:0.028).

Nuclear data of the present study:

Table (4): Defect size data of the present study:

\begin{tabular}{|l|l|l|l|}
\hline & Mean \pm SD & Range & Median \\
\hline Infarction size & $28 \pm 12.3$ & $6-61$ & 24 \\
\hline
\end{tabular}

The minimum defect size measured post-PCI was 6 and the maximum was 61 with mean value of 28 and SD 12.3.

Results of our study showed significant difference between patients with MACE or heart failure versus those without MACE or heart failure, regarding their defect size; $36.6 \pm 13.2$ versus $25.5 \pm 10.9$, (P value $\mathbf{0 . 0 0 5})$. The present study revealed statistically significant relationship between Infarction size and systolic function of the left ventricle estimated by the ECHO (P: 0.008).

Discussion:

The aim of this study is to assess the value of culprit vessel diameter using the simple index provided by the QCA method in the cath lab, in the setting of primary PCI, in predicting the myocardial infarction size, systolic function of the heart and inhospital clinical outcome .

Regarding clinical outcome in our study, 8 patients had heart failure $(15.4 \%)$ and
4 patients died eventually (7.7\%) with LAD culprit group had higher adverse clinical outcome than non-LAD culprit group (p:0.001).

Few studies were found to assess the value of culprit vessel diameter as a predictor of infarction size.

Our study was compatible with the following studies:

A ğaçet ${ }^{(12)}$ carried out investigations on 123 patients with their first attack of acute anterior STEMI and cocluded that the simple index derived from coronary angiography at the time of primary percutaneous coronary intervention can predict LV systolic function loss and adverse clinical outcome in patients with acute anterior MI. Also our study agreed with:

Gibbon (13) who concluded correlation between infarct size and mortality; and, demonstration that therapies which result in smaller infarct size also result in better clinical outcome in the same patients.Infarct size and myocardial salvage are the most extensively validated and widely applied surrogate endpoints in the setting of acute MI.

Conclusion: A simple index measured by QCA analysis in the setting of primary PCI is significantly correlated with loss of systolic function of the heart and adverse clinical outcome.

\section{References:}

1. Reimer KA (1979): The "wavefront phenomenon" of myocardial ischemic cell death. Transmural progression of necrosis within the framework of ischemic bed size (myocar- dium at risk) and collateral flow. Lab Invest .,40:633-44.

2. Christian TF (1992): Schwartz RS, Gibbons RJ. Determinants of infarct size

reperfusion therapy for acute myocardial infarc- tion. Circulation ,86:81-90.

3. Lee JT (1981): Myocardial infarct size and location in relation to the coronary vascular bed at risk in man. Circulation, 64:526-34

4. Andersen HR (2003): A comparison of coronary angioplasty with fibrinolytic therapy in acute 
myocardial infarction. New England journal of medicine, 733-742.

5. Betriu A (2005): Comparison of mortality rates in acute myocardial infarction treated by percutaneous coronary intervention versus fibrinolysis. Am J Cardiol., 95:100101.

6. Burns RJ (2002): The relationships of left ventricular ejection fraction, endsystolic volume index and infarct size to six-month mortality after hospital discharge following myocardial infarction treated by thrombolysis. J Am Coll Cardiol., 39(1):30-36.

7. Saran RK (1990): Reduction in STsegment elevation after thrombolysis predicts either coronary reperfusion or preservation of left ventricular function. Br Heart J., 64:113-117.

8. Allman KC (2002): Myocardial viability testing and impact of revascularization on prognosis in patients with coronary artery disease and left ventricular dysfunction: a meta-analysis. J Am Coll Cardiol ., 39:1151.

9. Senior $R$ (2002): Impact of revascularization and myocardial viability determined by nitrateenhanced Tc-99m sestamibi and Tl201 imaging on mortality and functional outcome in ischemic cardiomyopathy. J Nucl Cardiol ., 9:454.

10. Abd El-Fattah A (2006): Prognostic implication of normal exercise tomographic thallium imaging in patients with angiographic evidence of significant coronary artery disease. Am J Card ., 74:8, 769-71.

11. Gallik DM (1995): Simultaneous assessment of myocardial perfusion and left ventricular function during transient coronary occlusion. J Am Coll Cardiol. , 25: 1529-1538

12. Ağaçet M (2018). A simple index to predict adverse clinical outcome in acute STEMI in the primary PCI . www.onlinejacc.org/content/62/18_Su pplement_2/C4.2

13. Gibbon RJ(1989):Feasibility of tomographic Tc-99m-hexakis-2methoxy-2-methylpropyl-isonitrile imaging for the assessment of myocardial area at risk and the effect of treatment in acute myocardial infarction. Circulation, 80:1277-1286. 